Int. J. Dev. Biol. 61: 459-463 (2017)

doi: $10.1387 / \mathrm{ijdb} .160175 \mathrm{sj}$

\title{
DYRK2 displays muscle fiber type specific function during zebrafish early somitogenesis
}

\author{
WEI SUN ${ }^{1,2, \#, \text { SHUANG JIAO }{ }^{1,2, \#,} \text { XUNGANG TAN }{ }^{1,2, *}, \text { PEIJUN ZHANG }{ }^{1,2} \text { and FENG YOU }}{ }^{1,2}$ \\ ${ }^{1}$ Key Laboratory of Experimental Marine Biology, Institute of Oceanology, Chinese Academy of Sciences and \\ ${ }^{2}$ Laboratory for Marine Biology and Biotechnology, Qingdao National Laboratory for Marine Science and Technology, \\ Qingdao, Shandong, People's Republic of China
}

\begin{abstract}
Dual specificity tyrosine-phosphorylation regulated kinase 2 (DYRK2) is a serine/ threonine kinase. In zebrafish, DYRK2 is expressed in the lateral somites and adaxial cells at the early stage of embryo development. However, its role in early myogenesis had not been elucidated yet. Here, we report that DYRK2 mRNA and MyoD mRNA were colocalized in the muscle progenitor cells in somites, including both the posterior compartment of the lateral somites and adaxial cells. Knockdown of DYRK2 reduced the levels of $M y O D$ transcripts in the muscle progenitor cells in somites. In contrast, overexpression of DYRK2 increased the levels of MyoD transcripts in the muscle progenitor cells in somites. The effects of knockdown and overexpression of DYRK2 on the expression of $M y o D$ in the posterior compartment of the lateral somites were much greater than in the adaxial cells. Further studies indicated that forced expression of DYRK2 increased the levels of fast-twitch skeletal myosin RNA. Moreover, knockdown or forced expression of DYRK2 affected the levels of fast-twitch skeletal myosin protein. Together, these data indicate that DYRK2 is expressed in the developing muscle progenitor cells in somites and that it positively regulates fast-twitch muscle differentiation, at least at the early stages.
\end{abstract}

KEY WORDS: zebrafish, DYRK2, in situ hybridization, muscle development

Skeletal muscle is a major component of vertebrate anatomy. Vertebrate skeletal muscle is composed of two major types of muscle fibers that are functionally adapted through differences in their physiological and metabolic properties, namely fast-twitch muscle fibers and slow-twitch muscle fibers. Most mammalian skeletal muscles are heterogeneous in composition, containing both fast-and slow-twitch fibers and are capable of performing various functions (Schiaffino and Reggiani, 2011). By contrast, zebrafish embryonic myotome shows a discrete temporal and spatial separation of fiber type ontogeny, providing an excellent model for studying the molecular mechanisms involved in fiber type specification (Jackson et al., 2015). Zebrafish myogenesis begins prior to somite formation with the activation of the myogenic regulatory factors (MRFs), MyoD and Myf5 (Coutelle et al., 2001; Weinberg et al., 1996). During segmentation period, $M y o D$ transcripts are specifically expressed both in the adaxial cells and the posterior compartment of the lateral somites, which will differentiate into slow- and fast-twitch muscle fibers, respectively (Ochi and Westerfield, 2007; Weinberg et al., 1996).

Dual specificity tyrosine(Y)-phosphorylation regulated kinase 2
(DYRK2) is a member of the DYRK family that belongs to the CMGC group of protein kinases (Nihira and Yoshida, 2015). DYRK2 consists of a conserved kinase domain and an adjacent $\mathrm{N}$-terminal DYRK homology box. DYRK2 autophosphorylates a critical tyrosine residue in its own activation loop of the kinase domain and phosphorylates its substrates on serine and/or threonine residues. Once DYRK2 is fully translated and released from the ribosome, the transitional tyrosine kinase activity is lost and it then functions only as a serine/threonine kinase (Lochhead et al., 2005). DYRK2 is involved in regulating key developmental and cellular processes, such as neurogenesis, cell proliferation, cytokinesis, and cellular differentiation (Taira et al., 2007; Woods et al., 2001; Yoshida, 2008).

Our previous study have showed that zebrafish DYRK2 was specifically expressed in the lateral somites and adaxial cells that will develop into muscles (Sun et al., 2010), indicating that DYRK2 may

\footnotetext{
Abbreviations used in this paper: DYRK2, dual specificity tyrosine-phosphorylation regulated kinase 2 .
}

\footnotetext{
*Address correspondence to: Xungang Tan. Institute of Oceanology, Chinese Academy of Sciences, 7 Nanhai Road, Qingdao 266071, P.R. China. Tel: +86-532-8289-8551. E-mail: tanx@qdio.ac.cn (iD) http://orcid.org/0000-0001-7941-617X

\#The indicated authors contributed equally.
} 

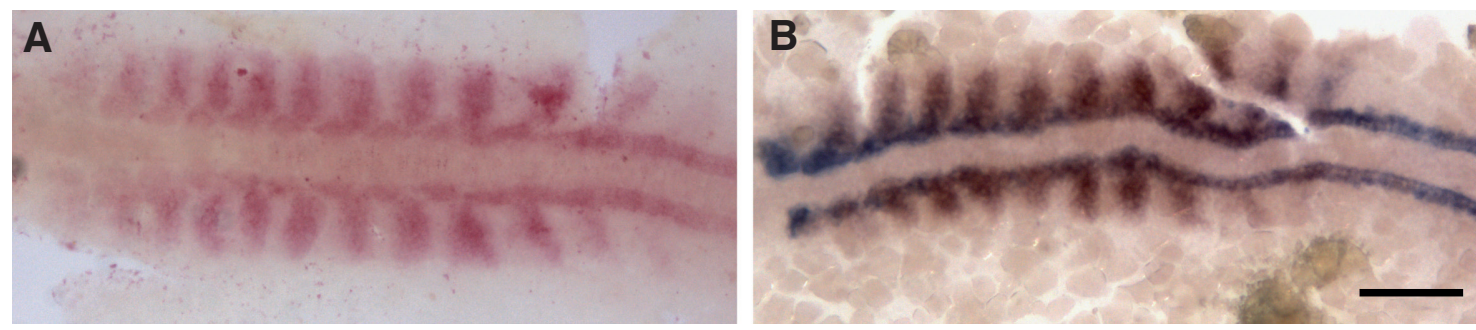

Fig. 1. One and two-color in situ hybridization in zebrafish embryos showing DYRK2 (blue) and MyoD (red) expression. Zebrafish embryos at the 11-somite stage. Head to the left, dorsal view. (A), One-color in situ hybridization showing MyoD expression in the posterior compartment of the lateral somites and adaxial cells. (B), Two-color in situ hybridization of DYRK2 and MyoD showing DYRK2 also expression in the posterior compartment of the lateral somites and adaxial cells. Scale bar, 10um.

be involved in the early development of muscle fiber specialization. However, the role of $D Y R K 2$ in this process remains unclear. In this study, we investigated the role of DYRK2 in regulating MyoDexpression, and its effects on fast-twitch muscle differentiation, in zebrafish embryos. We showed that DYRK2 mRNA and MyoD mRNA were colocalized in the muscle progenitor cells in somites, and DYRK2 was a positive regulator of $M y o D$ transcripts. Our further analyses revealed that DYRK2 also contributed to fast-twitch muscle fibers differentiation both at the mRNA and protein level. Our findings implied that DYRK2 was a key factor regulating muscle development at the early stage of embryo development.

\section{Results}

\section{ZebrafishDYRK2 mRNA and MyoD mRNAare colocalized in the} muscle progenitor cells in somites during early embryogenesis

Our previous study has shown that zebrafish DYRK2 mRNA was expressed in somite cells that will develop into muscles (Sun et al., 2010). Based on the anatomical location and distribution of the hybridization signal and published literature (Maves et al., 2007), we speculated that the DYRK2-expressing cells were located in the lateral somites and adaxial cells. To test this idea, we performed one-color (Fig. 1A) or two-color (Fig. 1B) in situ hybridization analysis using digoxigenin-labelled DYRK2 probe and fluorescein-labelled $M y o D$ probe. As shown in Fig. 1B, DYRK2mRNAsignals colocalized perfectly with MyoDmRNA signals, suggesting that the DYRK2gene is specifically expressed in the muscle progenitor cells in somites including both the posterior compartment of the lateral somite and adaxial cells.

Knockdown of DYRK2 reduces levels of MyoD transcripts in the muscle progenitor cells in somites during early embryogenesis

To investigate the possible role of endogenous DYRK2 in embryonic myogenesis, morpholino-mediated knockdown of $D Y R K 2$ was
A

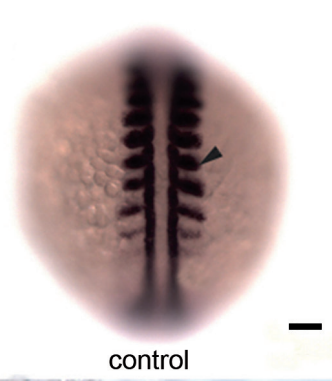

B

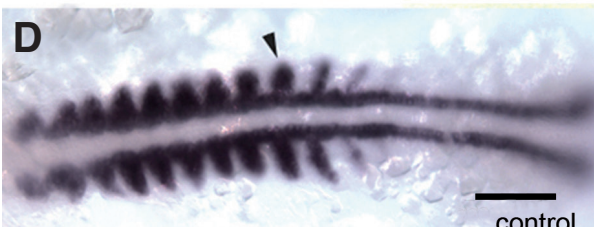

E

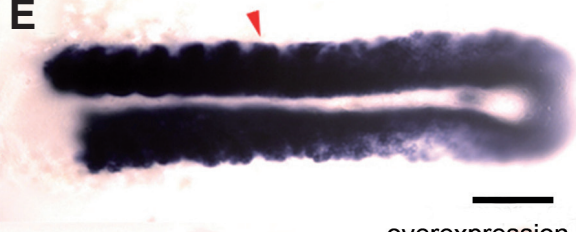

overexpression

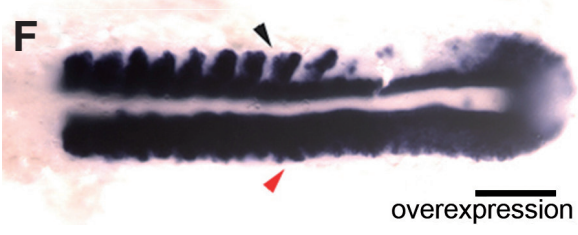

C
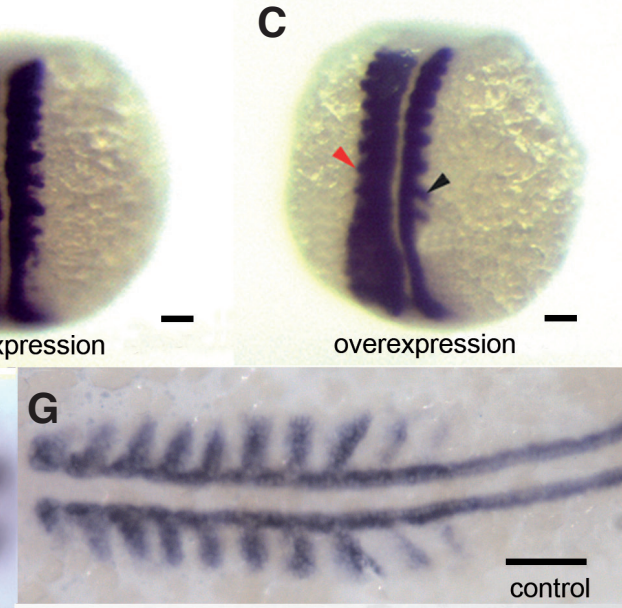

H

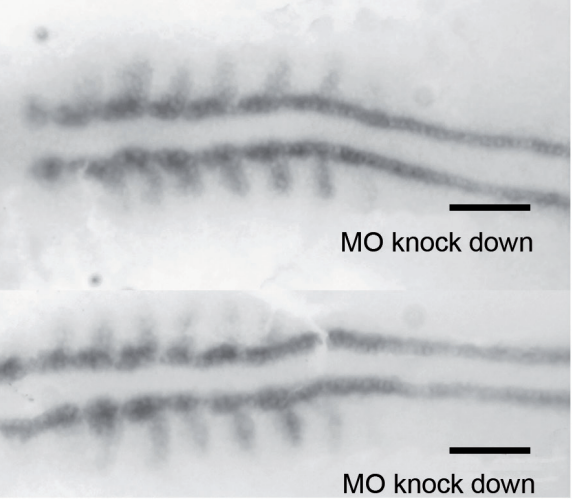

Fig. 2. Effect of DYRK2 forced expression and knockdown on the expression of $M y o D$ mRNA in zebrafish embryos. Zebrafish embryos at the 10-13-somite stage. (A-C) Dorsal views with anterior to the top. (D-I) Dorsal views with anterior to the left. $(\mathbf{A}, \mathbf{D})$ Control GFP mRNA-injected embryos. (G) A control MO-injected embryo. (A,D,G), MyoD expression in the posterior compartment of the lateral somites and adaxial cells. (B,E) MyoD mRNA showed increased expression in the posterior compartment of the lateral somites and adaxial cells from the front to back on both sides when DYRK2 mRNA was injected into one-cell stage embryos. (C,F) MyoD mRNA showed high-level expression at the side injection side when DYRK2 mRNA was injected into one of the two cells at the two-cell stage embryos. $(\mathbf{H}, \mathbf{I})$, Konckdown of DYRK2 by ATG-MO attenuated MyoD expression in the posterior compartment of the lateral somites and adaxial cells. The red and black arrowheads indicate the injected and uninjected side of embryos respectively. Scale bar, $10 \mu \mathrm{m}$. 
A

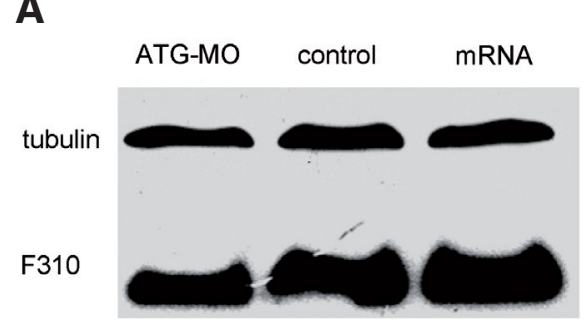

B

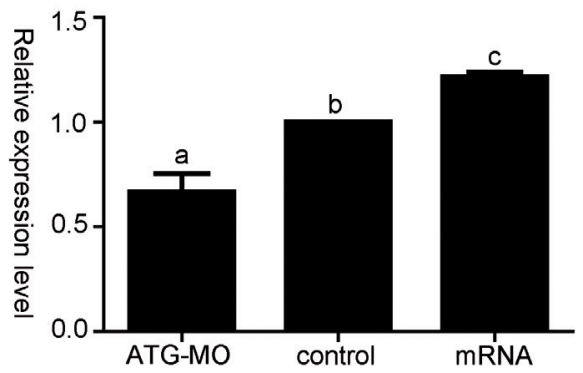

Fig. 3. Effect of DYRK2 knockdown and forced expression on the expression of fast-twitch skeletal myosin in zebrafish embryos analyzed by Western immunoblot. Embryos injected with ATG-MO or mRNA of DYRK2 displayed a decreased and an increased expression level of fast-twitch skeletal myosin. (A) Western immunoblot analysis of F310 and $\gamma$-tubulin indicated, Images were representative images from three independent experiments. (B) The relative expression levels of F310 were determined by densitometry and normalized by the $\gamma$-tubulin protein levels. Control group was set as 1. Data shown are means $\pm S E M, n=3$. Different letters represent statistically significant differences ( $p<0.05)$.

carried out. The specificity and efficacy of MOs were verified (data not shown). At 10-13-somite stage, MOs injected embryos were collected for in situ hybridization analysis of MyoD. As shown in Fig. 2 G-I, knockdown of $D Y R K 2$ decreased MyoD expression levels in both the lateral somites and adaxial cells, and the effect in former one was more dominant.

\section{Overexpression of DYRK2 increases levels of MyoD tran- scripts in the muscle progenitor cells in somites during early embryogenesis}

We next analyzed the effect of DYRK2 ectopic expression on embryonic myogenesis by in situ hybridization using $M y o D$. Ectopic expression of $D Y R K 2$ caused marked changes in MyoDexpression patterns between the GFP mRNA-injected control and DYRK2 mRNA injected embryos. As shown in Fig. 2 A,B,D,E, injection of $D Y R K 2$ mRNA at the one-cell stage resulted in robust expression of $M y O D$ in the developing somite cells including both the lateral

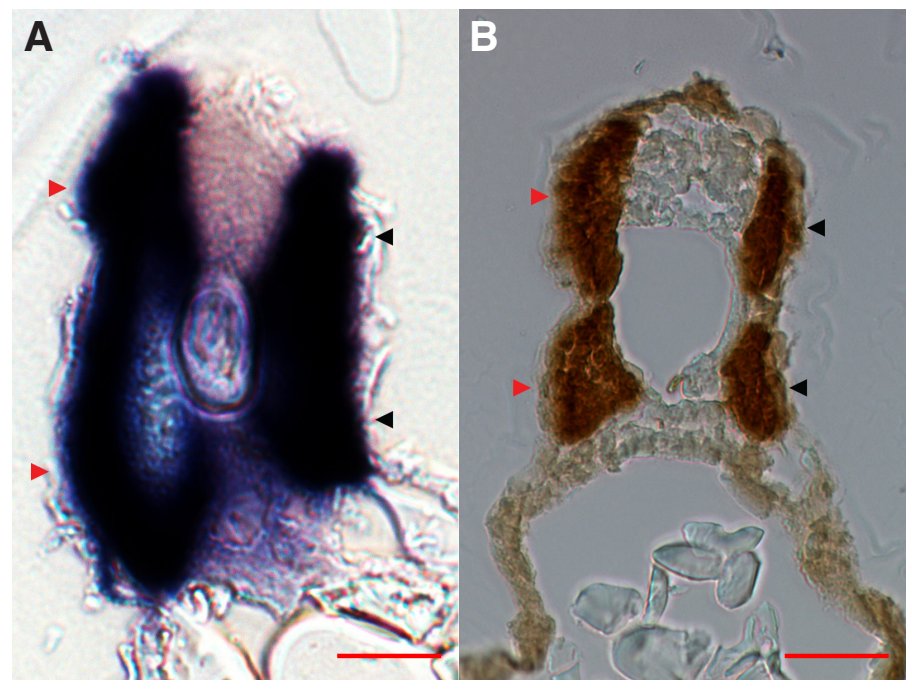

Fig. 4. Effect of DYRK2 forced expression on the expression of fast-twitch skeletal myosin both at the mRNA and protein level. DYRK2 mRNA was injected into one of the two cells at the two-cell stage embryos, and at 24hpf, some embryos were fixed and analyzed by in situ hybridizaition using the antisense probes against zebrafish fast muscle myosin heavy chain2 (myhz2) (Du et al., 2008) (A); and at 30hpf, some embryos were fixed and analyzed by immunostaining using the F310 anti-fast-twitch skeletal myosin light chain antibody (B). Transverse cryosections have dorsal towards the top. The red and black arrowheads indicate the injected and uninjected side of embryos respectively. Scale bar, $20 \mu \mathrm{m}$. somites and adaxial cells. Further, injection of DYRK2 mRNA in one cell at the two-cell stage only resulted in robust expression of $M y o D$ in the injected side of the developing somites (Fig. 2 A,C,D,F). Moreover, the effects of overexpression of DYRK2 on the expression of $M y O D$ in the lateral somites were much greater than that in the adaxial cells.

\section{DYRK2 regulates the expression of myosin in fast-twitch muscles during early embryogenesis}

We next examined the effects of DYRK2 knockdown and forced expression on the fast-twitch skeletal muscle differentiation. Compared with the control group, embryos injected with DYRK2 MO had significantly lower fast-twitch skeletal myosin levels. Furthermore, forced expression of DYRK2 significantly increased fast-twitch skeletal myosin levels, indicating that DYRK2 played a role in fast-twitch skeletal muscle fiber differentiation (Fig. $3 \mathrm{~A}, \mathrm{~B})$. Further, injection of DYRK2 mRNA in one cell at the twocell stage obviously increased fast-twitch skeletal myosin mRNA (Fig. 4A) and protein (Fig. 4B) expression in the injected side of the developing somites.

\section{Discussion}

In this study, we have showed that zebrafish DYRK2 mRNA was specifically co-expressed with MyoD mRNA in the muscle progenitor cells in somites including both the posterior compartment of the lateral somites and adaxial cells during early embryogenesis, which were the fast- and slow-twitch muscle precursor cells, respectively. Moreover, we have proved that zebrafish DYRK2 positively regulated $M y O D$ gene expression and fast-twitch muscle fiber differentiation during embryonic myogenesis. These results provided the first in vivo evidence that the DYRK2 gene may play a critical role in the process of fast-twitch muscle differentiation during embryonic myogenesis.

MyoD is essential for initiating the skeletal muscle program in the embryos (Weintraub, 1993). In this paper, we found DYRK2 and MyoD mRNA co-localized in the posterior compartment of the lateral somites and adaxial cells in zebrafish embryonic myogenesis. Moreover, both of their expression occurs during zebrafish embryogenesis far in advance of any overt myogenic differentiation (Sun et al., 2010; Weinberg et al., 1996). Therefore, we speculated that DYRK2 was an important factor in regulating embryonic myogenesis.

Our loss- and gain-of-function analysis results provide strong evidence supporting the view that DYRK2 plays an important role in regulating embryonic myogenesis. Overexpression of a func- 
tional DYRK2 protein increased expression of $M y o D$ both in the posterior compartment of the lateral somites and the adaxial cells. It is of interest to note that the increased levels of $M y O D$ transcripts in the lateral somites was much higher than that in the adaxial cells, which implied that DYRK2 might play a much stronger role in embryological development of fast-twitch muscle than in the slow-twitch muscle. Furthermore, targeted knockdown of DYRK2 significantly reduced expression of $M y O D$ both in the lateral somites and the adaxial cells, and also the effect in the former one was much higher than that in the latter one. The knockdown data also support the point of view that DYRK2 had an important role in embryonic myogenesis, especially in embryological development of fast-twitch muscle. Further research showed that DYRK2 positively regulated fast-twitch skeletal myosin mRNA and protein expression levels. To our knowledge, this study is the first to provide in vivo evidence showing DYRK2 is a positive regulator of fast-twitch muscle development in a vertebrate embryo.

Protein kinase DYRK2 phosphorylates its substrates on serine/ threonine residues (Lochhead et al., 2005). Therefore, we speculate that DYRK2 might play a role in mediating embryonic myogenesis by phosphorylating one or some key factors of the differentiation of muscle and transformation between the fast- and slow-twitch muscle, such as Gli2, Pbx2, and Pbx4 (Katoh and Katoh, 2008; Maves et al., 2007; Varjosalo et al., 2008; Yao et al., 2013). Further studies on the searching for the phosphorylated substrates of DYRK2 would be more interesting.

In summary, we provide evidence that zebrafish DYRK2 is a key factor in muscle development during embryogenesis, especially in the fast-twitch muscle development. Zebrafish embryonic myotome shows a discrete temporal and spatial separation of fiber type ontogeny, providing an excellent model for studying the molecular mechanisms involved in fiber type specification. Our study has also presented the opportunity to further elucidate the mechanism and the signalling pathways by which DYRK2 regulates muscle development using this unique vertebrate model organism.

\section{Materials and Methods}

\section{Experimental animals}

Zebrafish (Danio rerio) were maintained on a 14-h light, 10-h dark cycle at $28^{\circ} \mathrm{C}$ and fed twice a day. Embryos were obtained by natural crossing. Fertilized eggs were raised in embryo medium at $28.5^{\circ} \mathrm{C}$ and staged according to the standard method (Kimmel et al., 1995). 2-Phenylthiourea (Sigma-Aldrich, USA) $0.003 \%$ (wt/vol) was added to the water in some experiments to prevent pigmentation. All experiments were conducted according to the regulations of local and central governments, and approved by the Institutional Animal Care and Use Committee of Institute of Oceanology, Chinese Academy of Sciences.

\section{Morpholino knockdown}

Morpholino antisense oligos (Gene Tools, USA) were synthesized. The translation blocker (ATG-MO) was based on a sequence near the ATG (in bold) start site. The splicing blocker (E2I2-MO) was based on the sequence at the exon 2 and intron 2 junctions: ATG-MO: 5'- GGTTTTCTGCTTAATATCATCATGG-3'; and E2I2-MO: 5'- GAACAGGAAGTCGGACTTACAGTGT -3'. Besides, a standard control MO (CMO): 5'-CCTCTTACCTCAGTTACAATTTATA-3' were used as control. Morpholino antisense oligos were dissolved in 1X Danieau buffer to a final concentration of $1 \mathrm{mM}$. Next, 1-2 $\mathrm{nl}$ (5-10 ng) was injected into each embryo. The specificity and efficacy of each of these $\mathrm{MO}$ sequences have been confirmed as reported previously (Tan et al., 2006).

\section{Overexpression of DYRK2}

A previous pBlueScript SK (Stratagene, USA) vector (Sun et al., 2010) containing the entire ORF of zebrafish DYRK2 was digested with Sal I and BamH I and subcloned into Sall and BamH I sites of pSP64 vector. Capped mRNA was synthesized with a mMESSAGE mMACHINE SP6 Kit (Ambion, USA) and linearized plasmid DNA as template. $2 \mathrm{nl}$ mRNA (25 ng/ $\mu l)$ was microinjected into one cell of zebrafish embryos at the one-cell or two-cell stage, respectively. GFP mRNA ( $25 \mathrm{ng} / \mu \mathrm{l})$ injected embryos were used as controls. After injection, embryos were placed in embryo rearing medium and kept at $28.5^{\circ} \mathrm{C}$.

\section{Whole-mount in situ hybridization and transverse cryosection}

One-color or two-color in situ hybridization using digoxigenin-labeled or fluorescein-labeled antisense riboprobes was performed as reported previously (Tan and Du, 2002). For the DYRK2 knockdown/ overexpression and Myod expression experiments, all of the treatments, including microinjection, fixation, in situ hybridization and revelation, were in parallel. For the DYRK2 overexpression and $\mathrm{FMHC}$ expression experiments, after whole-mount in situ hybridization, some embryos were proceed for transverse cryosection at $10 \mu \mathrm{m}$. pGEM-myhz2 plasmid for synthesizing fMHC antisense probe was kindly provided by Dr. Shao jun Du. Images were captured with a QImaging 5.0RTV CCD Camera mounted on an Olympus microscope (Olympus, Japan).

\section{Analysis of protein expression by Western blot in injected zebrafish embryos}

Wild-type embryos were injected with GFP or DYRK2 capped mRNA or DYRK2 ATG-MO at the one-cell stage. Injected embryos were analyzed by immunostaining by using the F310 anti-fast-twitch skeletal myosin light chain antibody (DSHB, USA) and anti- $\gamma$-tubulin antibody (Sigma, USA) at $30 \mathrm{hpf}$. Briefly, zebrafish embryos were dechorionated, deyolked, homogenized and boiled in $2 \times$ sodium dodecyl sulfate (SDS) lysis buffer $(125 \mathrm{mM}$ Tris- $\mathrm{HCl}$, pH6.8, 4\% SDS, 20\% glycerol, 10\% $\beta$-mercaptoethanol, $0.02 \%$ Bromophenol Blue) $(2.5 \mu$ l for each embryo) containing $10 \mu \mathrm{g} / \mathrm{ml}$ aprotinin, $10 \mu \mathrm{g} / \mathrm{ml}$ leupeptin, $10 \mu \mathrm{g} / \mathrm{ml}$ pepstatin, and 100 mM PMSF. The homogenates were collected by low speed centrifugation at $3000 \mathrm{rpm}$ for $1 \mathrm{~min}$ $10 \mu \mathrm{l}$ were loaded each lane on a $10 \%$ SDS-PAGE and then analyzed by Western immunoblot.

\section{Whole-mount immunostaining and transverse cryosection}

The whole mount immunostaining was carried out with the injected embryos at 30 hpf using the F59 anti-slow-twitch skeletal myosin heavy chain antibody (DSHB, USA), and the F310 anti-fast-twitch skeletal myosin light chain antibody (DSHB, USA) as previously described(Tan etal., 2006). Antimouse IgG secondary antibody was labeled with horseradish peroxidase (Invitrogen, USA). Following whole-mount immunostaining, some embryos were proceed for transverse cryosection at $10 \mu \mathrm{m}$.

\section{Acknowledgments}

This work was supported by the Shandong Provincial Natural Science Foundation, China (No. BS2014HZ008), the Natural Science Foundation of China (No. 31502146, No. 30970423, No. 31128017), the Scientific and Technological Innovation Project Financially Supported by Qingdao National Laboratory for Marine Science and Technology (No. 2015ASKJ02), and the National Infrastructure of Fishery Germplasm Resource (No. 2017DKA30470).

\section{References}

COUTELLE, O., BLAGDEN, C.S., HAMPSON, R., HALAI, C., RIGBY, P.W.J. and HUGHES, S.M. (2001). Hedgehog signalling is required for maintenance of myf5 and myoD expression and timely terminal differentiation in zebrafish adaxial myogenesis. Dev Biol 236: 136-150.

DU, S.J., LI, H.Q., BIAN, Y. and ZHONG, Y. (2008). Heat-shock protein 90 alpha 
1 is required for organized myofibril assembly in skeletal muscles of zebrafish embryos. Proc. Natl Acad Sci USA 105: 554-559.

JACKSON, H.E., ONO, Y., WANG, X.G., ELWORTHY, S., CUNLIFFE, V.T. and INGHAM, P.W. (2015). The role of Sox6 in zebrafish muscle fiber type specification. Skelet Muscle 5: 2.

KATOH, Y. and KATOH, M. (2008). Integrative genomic analyses on GLI2: Mechanism of Hedgehog priming through basal GLI2 expression, and interaction map of stem cell signaling network with P53. Int J Oncol 33: 881-886.

KIMMEL, C.B., BALLARD, W.W., KIMMEL, S.R., ULLMANN, B. and SCHILLING, T.F. (1995). Stages of Embryonic-Development of the Zebrafish. Dev Dynam 203: 253-310.

LOCHHEAD, P.A., SIBBET, G., MORRICE, N. and CLEGHON, V. (2005). Activationloop autophosphorylation is mediated by a novel transitional intermediate form of DYRKs. Cell 121: 925-936.

MAVES, L., WASKIEWICZ, A.J., PAUL, B., CAO, Y., TYLER, A., MOENS, C.B. and TAPSCOTT, S.J. (2007). Pbx homeodomain proteins direct Myod activity to promote fast-muscle differentiation. Development 134: 3371-3382.

NIHIRA, N.T. and YOSHIDA, K. (2015). Engagement of DYRK2 in proper control for cell division. Cell Cycle 14: 802-807.

OCHI, H. and WESTERFIELD, M. (2007). Signaling networks that regulate muscle development: Lessons from zebrafish. Dev Growth Differ 49: 1-11.

SCHIAFFINO, S. and REGGIANI, C. (2011). Fiber types in mammalian skeletal muscles. Physiol Rev 91: 1447-1531.

SUN, W., TAN, X.G., ZHANG, P.J., ZHANG, Y.Q. and XU, Y.L. (2010). Characterization of DYRK2 (dual-specificity tyrosine-phosphorylation-regulated kinase 2) from Zebrafish (Dario rerio). Chin J Oceanol Limn 28: 720-724.

TAIRA, N., NIHIRA, K., YAMAGUCHI, T., MIKI, Y. and YOSHIDA, K. (2007). DYRK2 is targeted to the nucleus and controls p53 via Ser46 phosphorylation in the apoptotic response to DNA damage. Mol Cell 25: 725-738.

TAN, X. and DU, S.J. (2002). Differential expression of two MyoD genes in fast and slow muscles of gilthead seabream (Sparus aurata). Dev Genes Evol212: 207-217.

TAN, X., ROTLLANT, J., LI, H., DE DEYNE, P. and DU, S.J. (2006). SmyD1, a histone methyltransferase, is required for myofibril organization and muscle contraction in zebrafish embryos. Proc Natl Acad Sci USA 103: 2713-2718.

VARJOSALO, M., BJORKLUND, M., CHENG, F., SYVANEN, H., KIVIOJA, T., KILPINEN, S., SUN, Z., KALLIONIEMI, O., STUNNENBERG, H.G., HE, W.W. et al., (2008). Application of active and kinase-deficient kinome collection for identification of kinases regulating hedgehog signaling. Cell 133: 537-548.

WEINBERG, E.S., ALLENDE, M.L., KELLY, C.S., ABDELHAMID, A., MURAKAMI T., ANDERMANN, P., DOERRE, O.G., GRUNWALD, D.J. and RIGGLEMAN, B. (1996). Developmental regulation of zebrafish MyoD in wild-type, no tail and spadetail embryos. Development 122: 271-280.

WEINTRAUB, H. (1993). The Myod Family and Myogenesis - Redundancy, Networks, and Thresholds. Cell 75: 1241-1244.

WOODS, Y.L., COHEN, P., BECKER, W., JAKES, R., GOEDERT, M., WANG, X.M. and PROUD, C.G. (2001). The kinase DYRK phosphorylates protein-synthesis initiation factor elF2B epsilon at Ser(539) and the microtubule-associated protein tau at Thr(212): potential role for DYRK as a glycogen synthase kinase 3-priming kinase. Biochem J 355: 609-615.

YAO, Z.Z., FARR, G.H., TAPSCOTT, S.J. and MAVES, L. (2013). Pbx and Prdm1a transcription factors differentially regulate subsets of the fast skeletal muscle program in zebrafish. Biol Open 2: 546-555.

YOSHIDA, K. (2008). Role for DYRK family kinases on regulation of apoptosis. Biochem Pharmacol 76: 1389-1394. 


\section{Further Related Reading, published previously in the Int. J. Dev. Biol.}

SMYD2 is induced during cell differentiation and participates in early development

Borja Sesé, Maria J. Barrero, Maria-Carme Fabregat, Veronika Sander and Juan Carlos Izpisua Belmonte

Int. J. Dev. Biol. (2013) 57: 357-364

http://dx.doi.org/10.1387/ijdb.130051ji

Cytoskeletal heart-enriched actin-associated protein (CHAP) is expressed in striated and smooth muscle cells in chick and mouse during embryonic and adult stages

Willemijn van Eldik, Abdelaziz Beqqali, Jantine Monshouwer-Kloots, Christine Mummery and Robert Passier

Int. J. Dev. Biol. (2011) 55: 649-655

http://dx.doi.org/10.1387/ijdb.103207wv

A critical role for myoglobin in zebrafish development

Danielle H. Vlecken, Janwillem Testerink, Elisabeth B. Ott, Philippe A. Sakalis, Richard T. Jaspers and Christoph P. Bagowski

Int. J. Dev. Biol. (2009) 53: 517-524

http://dx.doi.org/10.1387/ijdb.082651dv

Zebrafish spata2 is expressed at early developmental stages

Enrico Moro, Claudio Maran, M. Liliana Slongo, Francesco Argenton, Stefano Toppo and Maurizio Onisto

Int. J. Dev. Biol. (2007) 51: 241-246

http://dx.doi.org/10.1387/ijdb.062220em

Spatiotemporal expression of the creatine metabolism related genes agat, gamt, and ct1 during zebrafish embryogenesis

Lifeng Wang, Ying Zhang, Ming Shao and Hongwei Zhang

Int. J. Dev. Biol. (2007) 51: 247-253

http://dx.doi.org/10.1387/ijdb.062218lw

5 yr ISI Impact Factor $(2013)=2.879$
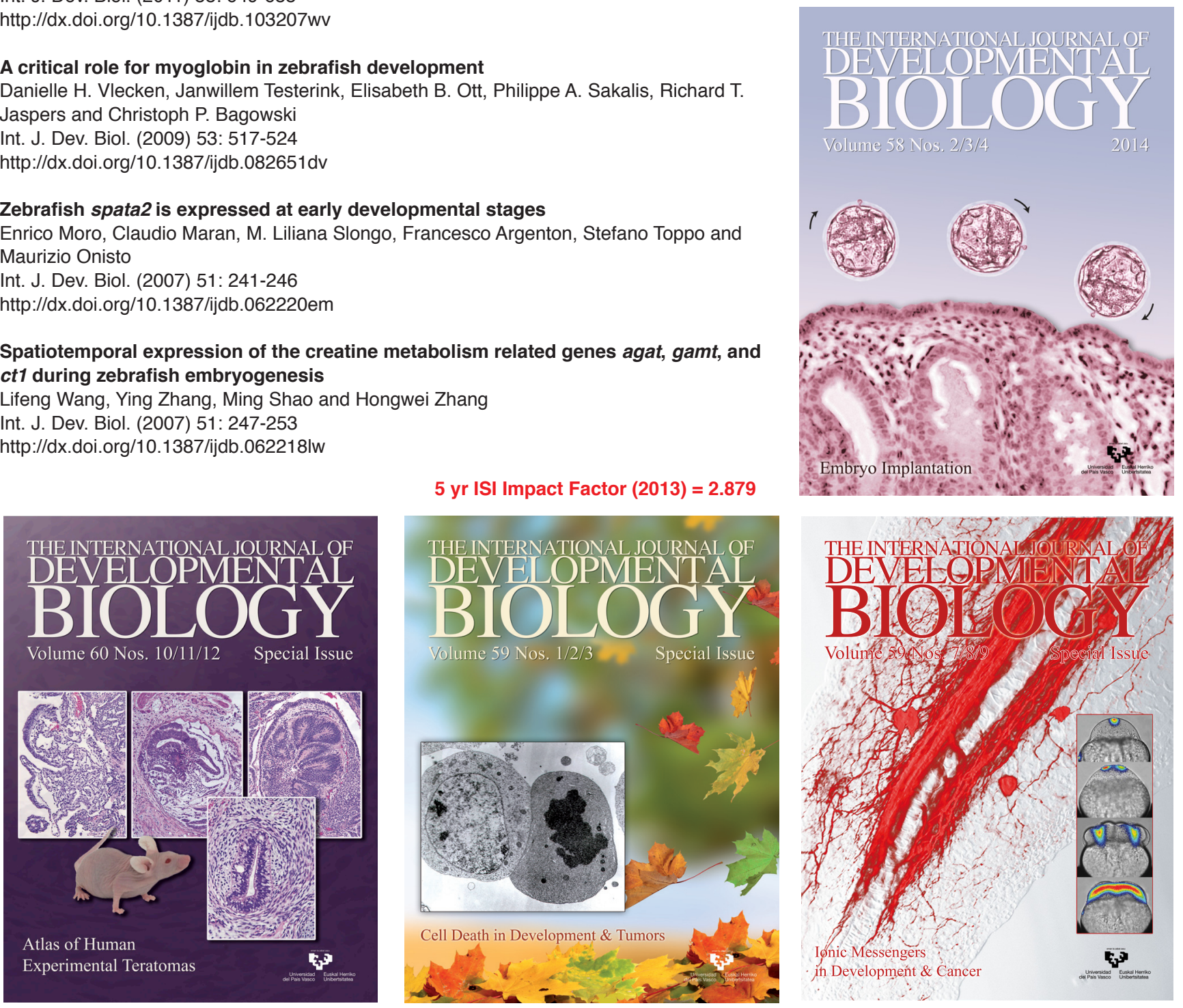